Revista de Psicología de la PUCP. Vol. XVII, 1, 1999.

\title{
IMPACTO DE LA EDUCACIÓN INICIAL EN EL RENDIMIENTO EN PRIMER GRADO DE PRIMARIA EN ESCUELAS PÚBLICAS URBANAS DE LIMA ${ }^{1}$
}

\author{
Santiago Cueto² y Juan José Díaz
}

Uno de los problemas más importantes de la educación en el Perú es la baja eficiencia de la primaria pública. En el presente artículo se analiza el impacto de dos tipos de programas de educación inicial pública, los Centros de Educación Inicial (CEl) y los Programas No Escolarizados de Educación Inicial (PRONOEI). Para ello se urilizó una encuesta retrospectiva, realizada en nueve escuelas públicas de Lima que mostraron altas tasas de repitencia. Entre aquellos niños que pasaron por un CEI, la probabilidad de obtener un resultado satisfactorio en lenguaje aumentó en $25 \%$, mientras que en matemática aumentó en 22\%. Para los niños que pasaron por un PRONOEI, estas probabilidades aumentaron en 15 y $17 \%$ en lenguaje y matemática, respectivamente.

Palabras claves: pre-escolaridad, rendimiento, primer grado, escuela pública, repitencia.

The impact of preschool programs in the achivement of first graders in elementary public schools of Lima

One of the major educational problems in Peru is the low level of efficiency attained in elementary schools. In this paper we analyze the impact of two types of preschool programs, Centros de Educacion Inicial (CEI) and Programas No Escolarizados de Educación Inicial (PRONOEI). We carried out a retrospective survery in nine public schools in Lima that had a high repetition rate. Among children with preschool education at CEI the probability of getting a higher achievement increases in $25 \%$ in language and $22 \%$ in mathematics. Amog children with preschool education at PRONOEI, the probability of obtaining a higher achievement increases in 15\% in language and $17 \%$ in mathematics. Key words: preschool, achicvement, first grade, public school, repetition.

1. La presente investigación fue posible gracias al apoyo de la Dirección Académica de Investigación de la PUCP. Milagros Arakaki, Fabiola Chávarry, Carola Flores, Giovanna Moreano, Jorge Pérez Reyes, Luz Elena Pinto y Nérida Urcia colaboraron en la recolección de datos. Una versión preliminar se presentó en el Seminario "El Desarrollo Infantil: una estrategia para el cambio social en el próximo milenio".

2. Realizó sus estudios de psicología en la PUCP. Obtuvo el doctorado en la Universidad de Indiana. Actualmente se desempeña como investigador de GRADE y es profesor de psicología en la PUCP. CE: scueto@grade.org.pe

3. Es Bachiller en economía por la PUCP; actualmente es asistente de investigación en GRADE. 



\section{Antecedentes}

Uno de los problemas principales del sistema educativo peruano es la baja eficiencia a nivel de la primaria, especialmente en primer grado. Sólo el $65.1 \%$ de los niños matriculados en 1992 se matricularon en segundo grado el siguiente año, $19.7 \%$ repitieron $^{4}$ y $15.2 \%$ abandonaron (Ministerio de Educación, 1993). Estas cifras son de lejos las peores para cualquier grado del sistema educativo peruano. Este problema es común a Latinoamérica, donde la repetición ha sido identificada como uno de los problemas cruciales en educación (Schiefelbein y Wolfff, 1993). Los altos índices de repetición generan gastos que han sido estimados en 4.2 millones de dólares anuales para la región (Schiefelben y Wolff, 1993). Sin embargo, en el Perú existen pocos estudios empíricos sobre los factores asociados a esta baja eficiencia y en cambio hay mucha especulación. Entre los múltiples motivos de bajo rendimiento en primer grado podría estar una pobre o inexistente preparación para la escuela. La evidencia empírica a nivel internacional muestra que la educación inicial es una de las estrategias para aumentar la eficiencia escolar (Myers, 1992, 1996; Schiefelbein, 1990). En el presente estudio no se cuestionan estos resultados, sino que se busca evaluar el impacto de los programas públicos tal cual son implementados actualmente en el Perú. Específicamente se analizará el impacto del paso de estudiantes por uno de dos tipos de programas públicos de educación inicial: los Centros de Educación Inicial (CEI) y los Programas No Escolarizados de Educación Inicial (PRONOEI), contrastándolo en cada caso con el de estudiantes que no hayan tenido educación inicial.

4. A partir de 1997 no hay repitencia en primer grado por disposición del Ministerio de Educación. La repitencia ha sido postergada hasta el final de cada ciclo (segundo, cuarto y sexto grados de primaria) 
Tanto los PRONOEI como los CEI son instituciones estatales orientadas a niños entre 3 y 5 años. Ambos buscan el desarrollo integral del niño a través de actividades de estimulación (cognitiva, motora, afectiva y social). La diferencia entre ambos radica en que los PRONOEI suelen estar a cargo de madres de familia de la comunidad (llamadas "animadoras" o "promotoras" 5 . Las animadoras reciben una capacitación inicial y luego son supervisadas periódicamente (idealmente una vez por semana son visitadas en su PRONOEI) por profesoras tituladas (llamadas "docentes coordinadoras"). Además los PRONOEI se forman en base a la participación de la comunidad, que tiene que organizarse y designar un local para el PRONOEI y recomendar a la animadora. Las animadoras reciben una "propina” de parte del Estado (S/. 200 mensuales; Data Social, 1998), sin embargo esta propina suele demorarse (Ministerio de Educación, 1996). Los CEI en cambio suelen estar a cargo de maestras tituladas que reciben un sueldo variable (en todo caso mayor al que reciben las animadoras). Los CEI funcionan en locales estatales especialmente construidos en la mayoría de los casos. Una última diferencia es que los CEI suelen tener clases 5 días a la semana, mientras que los PRONOEI suelen tener 4 (dedicando el quinto a la supervisión de las labores). Los PRONOEI resultan menos costosos para el Estado que los CEI y se han orientado sobre todo a zonas rurales y urbano-marginales de todo el Perú.

Aunque ninguno de los programas de educación inicial mencionados busca solamente preparar al niño para la escuela (esto explica por qué muchos autores prefieran el término "educación inicial" sobre "educación pre-escolar"), uno de los beneficios de estos programas debería ser una mejor escolaridad. Este estudio presenta datos al respecto. En la investigación no se incluirán centros de educación privada por que sólo representan el 16\% del estudiantado de primaria a nivel nacional (Ministerio de Educación, 1993). Por otro lado, los problemas más graves de rendimiento escolar se presentan en escuelas públicas (ver por ejemplo Cueto, Jacoby y Pollitt, 1997).

5. No es raro sin embargo encontrar un PRONOEI donde la profesora es una estudiante de educación y no una madre de la comunidad. 
La educación inicial es el sector educativo de más rápido crecimiento en el Perú y, de acuerdo a la Constitución del Perú de 1993, es obligatoria para los niños de 5 años. Sin embargo, la cobertura es aun baja en comparación a primaria, aunque ha aumentado rápidamente en los últimos años. De los niños que tienen entre 3 y 5 años de edad, en 1980 el 15\% asistían a un centro de educación inicial. Para 1997 la cifra es 62\% (datos proporcionados por el Ministerio de Educación; Data Social, 1998). En contraste, el porcentaje de niños entre 6 y 11 años que asiste a la escuela es $95 \%$ (El Peruano, 1998). El Ministerio de Educación se ha propuesto una cobertura del $100 \%$ de los niños entre 4 y 5 años para el 2007 (Ministerio de Educación, 1997), aunque no existe una política definida de hacerlo en CEI, PRONOEI o ambos ${ }^{6}$.

\section{Estudios previos}

A nivel internacional existen una serie de estudios que demuestran la importancia de la educación inicial para el posterior rendimiento escolar. Entre ellos destacan los estudios realizados sobre Head Start y el proyecto del Perry Preschool, en especial por que poseen datos sobre los efectos a largo plazo.

El programa Head Start es tal vez uno de los programas más grandes de educación pre-escolar a nivel mundial. Se inició a principios de los años 60 en los Estados Unidos, y con el se buscaba ayudar a niños provenientes de familias de escasos recursos para que ingresen mejor preparados a la escuela primaria. Los estudios iniciales mostraron un efecto positivo en el desarrollo intelectual al ingreso a la escuela y durante al menos los primeros tres años de escolaridad (Myers, 1992). Sin embargo se especula aun sobre los efectos a largo plazo de Head Start o los efectos para diferentes subgrupos (por ejemplo de acuerdo a la calidad del centro educativo al que asistan; Currie y Thomas, 1998).

6. Actualmente los PRONOEI tienen aproximadamente al $35 \%$ de los matriculados y los CEI al 65\% (INANDEP, 1993). 
El Perry Preschool Project constituye probablemente el estudio a largo plazo más ambicioso de los Estados Unidos. Este proyecto estuvo dirigido a niños urbanos negros de familias de escasos recursos económicos de Michigan. Los resultados de una muestra de estos estudiantes demostró que, a los 19 años, las probabilidades de graduarse y conseguir empleo eran mayores que las de una muestra de jóvenes de similar origen que no participó en el Proyecto. Adicionalmente, los que participaron en el proyecto requirieron menos educación remedial, quedaron embarazadas como adolescentes menos frecuentemente y tuvieron menos problemas con la ley (Myers, 1992).

En el Perú sólo conocemos de un estudio empírico sobre el impacto de la educación inicial en el rendimiento escolar. Este estudio fue realizado por Myers (1992) en Puno, comparando a niños que pasaron por un PRONOEI con niños que no tuvieron educación inicial. Los niños de PRONOEI obtuvieron mejores puntajes en una prueba estandarizada de desarrollo intelectual, motor y social. Sin embargo, no se encontraron diferencias en las tasas de promoción de primer a segundo ni de segundo a tercer grado. Entre las deficiencias que nota Myers sobre los PRONOEI, están las escasas habilidades pedagógicas de las animadoras, el tiempo empleado en actividades educativas en el aula, la disponibilidad y uso de materiales y la escasa supervisión de la labor de las animadoras.

El objetivo general del presente estudio es determinar el impacto de la educación inicial pública en el rendimiento escolar en alumnos de primer grado de centros educativos considerados de bajo rendimiento. Educación inicial pública se definirá como el paso por un CEI o PRONOEI entre los 3 y 5 años (comparado con no tener educación inicial previa a la matrícula en primer grado). El rendimiento escolar en este estudio se medirá a través de las calificaciones otorgadas por el profesor en comunicación integral (o lenguaje) y lógico-matemática.

\section{Hipótesis}

La hipótesis general de este estudio es que aquellos niños que pasen por educación inicial tendrán mejor rendimiento en primer grado que 78 
Impacto de la educación inicial en el rendimiento en primer grado de primaria...

aquellos que nunca la tuvieron. A nivel específico esperamos probar que los niños que hayan pasado por un CEI tendrán mejor rendimiento que aquellos que hayan pasado por un PRONOEI, quienes a su vez tendrán mejor rendimiento que aquellos que no hayan pasado por ningún centro de educación inicial. La hipótesis respecto a la diferencia entre CEI y PRONOEI no se basa en estudios previos (que no existen) sino en el hecho de que los CEI son atendidos por profesoras con título y los PRONOEI por madres con una capacitación de corta duración y con supervisiones periódicas. Es de suponer que una profesora licenciada tendrá mejores resultados con sus alumnos que una que no ha estudiado educación superior. Por otro lado, es común escuchar a especialistas en educación dudar de la calidad de la educación ofrecida por los PRONOEIS (ver por ejemplo comentarios de Myers mencionados antes).

\section{Metodología}

\section{Universo y participantes}

Se seleccionó 304 alumnos de 9 centros educativos públicos de los cuatro conos de la zona urbana de Lima ${ }^{7}$. De cada centro educativo se tomó un salón al azar. Los centros educativos fueron identificados con la asistencia del personal de la Unidad de Servicios Educativos (USE) local. Los colegios se elegieron con los siguientes criterios:

- Centros educativos que sean identificados en la USE cuyos alumnos presentan problemas bajo rendimiento ${ }^{8}$.

- Centros educativos que tengan en la comunidad tanto CEI como PRONOEI (de este modo queríamos comparar estudiantes del mismo

7. Los centros educativos pertenecían a las Unidades de Servicios Educativos 01 (San Juan de Miraflores), 04 (Comas), 06 (Vitarte), y Dirección de Educación del Callao.

8. En los últimos años el Ministerio de Educación ha administrado pruebas de rendimiento a nivel nacional. Lamenrablemente los resultados no han sido publicados. Estos hubieran permitido identificar más objetivamente los centros educativos de mas bajo rendimiento.Por ello se pidió a un especialista de primaria de cada USE que identifique centros educativos donde se dan mayores porcentajes de repitencia y deserción cn primer grado. 
medio socioeconómico y cultural; las diferencias entre estudiantes fueron controladas en el modelo estadístico explicado más adelante).

Todos los niños que cumplieron con los siguientes criterios fueron seleccionados:

- Ingresantes a primer grado por primera vez en 1997. No se consideró a repitentes en la medida en que tienen una historia educacional que difícilmente se puede comparar con la de los que ingresan por primera vez.

- No haber abandonado el primer grado en 1997 por motivos de salud, viaje, o alguna otra emergencia.

De acuerdo a lo anterior la muestra fue seleccionada intencionalmente para reflejar aquellos centros educativos urbanos de Lima con mayores problemas de repitencia y deserción. Sin embargo el número de centros educativos (nueve) es reducido, lo cual limita la validez externa de los resultados. La muestra total fue de 304 alumnos, $48 \%$ fueron hombres, con una edad promedio de 6 años al inicio del primer grado, véase el Cuadro 1.

\section{Cuadro 1}

\section{Caracteristicas de la Muestra}

\begin{tabular}{|c|c|c|c|c|c|}
\hline & \multirow{2}{*}{$\begin{array}{l}\text { Número } \\
\text { de casos } \\
(1)\end{array}$} & \multirow{2}{*}{$\begin{array}{c}\text { Edad } \\
\text { actual } \\
(2)\end{array}$} & \multirow{2}{*}{$\begin{array}{l}\text { Edad en } \\
\text { ler grado } \\
(3)\end{array}$} & \multicolumn{2}{|c|}{ Porcentaje que pasó por } \\
\hline & & & & CEI & PRONOEI \\
\hline Mujeres & 157 & 7.5 & 6.2 & $57.3 \%$ & $20.4 \%$ \\
\hline Hombres & 147 & 7.3 & 6.1 & $51.7 \%$ & $25.2 \%$ \\
\hline Total & 304 & 7.4 & 6.2 & $54.6 \%$ & $22.7 \%$ \\
\hline
\end{tabular}

Nota. (1) Se excluyeron ocho casos en los que no se completó la pregunta sobre el sexo del niño. (2) Corresponde a la edad del niño en el momento de la encuesta. (3) Corresponde a la edad del niño en el momento de ingresar al ler. grado. 
Impacto de la educación inicial en el rendimiento en primer grado de primaria...

\section{Variables $y$ mediciones}

La variable independiente es el paso por educación inicial, con tres niveles posibles: no o sí (PRONOEI o CEI).

Las variables dependientes fueron:

- Deserción: en este estudio se consideró deserción en un sentido amplio para incluir aquellos alumnos que abandonan la escuela por cualquiera de los siguientes motivos: expulsión del colegio por límite de inasistencias o decisión de los padres de retirar a su hijo(a) del colegio. Debemos decir sin embargo que no encontramos deserción en los centros educativos visitados (en otras palabras todos aquellos que estuvieron matriculados en 1997 en primer grado continuaban en la escuela en 1998). Este dato coincide con estadísticas reportadas recientemente, que indican que el $99 \%$ de los niños entre 6 y 11 años de Lima metropolitana están en la escuela (El Peruano, 1998).

- Por otro lado, se encontró estudiantes que habían repetido de grado aun a pesar de estar prohibido de acuerdo al Ministerio de Educación. Específicamente 5\% de los estudiantes en la muestra repitieron de grado. En la mayoría de casos el director o docente de aula había hablado con los padres de familia para explicarles que su hijo(a) no había aprendido lo suficiente $y$, por lo tanto, sería mejor que el siguiente año fuera matriculado por los padres en primer grado nuevamente (en algunos casos se pedía a los padres que el niño no asista los últimos meses del año para poder declarar que había abandonado y matricularlo nuevamente en primer grado el año siguiente).

- Notas escolares de fin de grado: en 1997 se implementó el nuevo currículo de educación primaria que instaura un sistema de calificación en base a letras: A (logró competencia), B (en proceso de lograr competencia) y C (no logró competencia) ${ }^{9}$. Se registrará la calificación

9. Se menciona a menudo que este nuevo sistema de calificación es "cualitativo", a diferencia del anterior sistema vigesimal que era "cuantitativo". Se debe señalar sin embargo que a las calificaciones $\mathrm{A}, \mathrm{B}$ y $\mathrm{C}$ subyace al menos una escala de medición ordinal $(\mathrm{A}>\mathrm{B}>\mathrm{C})$ que no puede ser considerada cualitativa. Una evaluación cualitativa requeriría una descripción única y detallada para cada 
asignada al final del año escolar en las áreas comunicación integral (antes lenguaje) y lógico-matemática (antes matemática).

Finalmente se registrarán datos sobre una serie de variables de control, que se pueden agrupar en:

- Variables del estudiante: se registró el sexo y si el estudiante trabajaba o no. Esta última variable no pudo ser introducida en el modelo por que no tuvimos suficientes casos de niños trabajadores de primer grado en la muestra. En otros contextos sin embargo se ha demostrado el efecto negativo de esta variable en el rendimiento escolar (ver por ejemplo Lockheed y Verspoor, 1991).

- Variables de la familia: se registró educación de la madre, número de personas por habitación (esta variable es referida como "hacinamiento" por el INEI, 1994; se considera hacinamiento cuando el número de personas por habitación excede 3).

- Variables de la escuela: registramos el centro educativo de cada estudiante para controlar por la variabilidad esperada entre los centros educativos.

\section{Métodos y técnicas}

Se desarrollaron dos encuestas, una sobre el centro educativo y otra sobre el estudiante para obtener los datos antes mencionados. Las encuestas recogen datos de los registros del centro educativo o de uno de los padres de familia de los estudiantes (en la mayoría de los casos se citó a los padres al centro educativo, de no asistir se les entrevistó en casa o el centro de trabajo). Todos los datos presentados en el estudio están referidos a características del estudiante y su entorno en 1997 y fueron tomados entre julio y setiembre de 1998.

estudiante en base a sus destrezas, limitaciones y potenciales. En todo caso es obvio que las evaluaciones cualitativas y las cuantitativas no tienen por qué ser consideradas opuestas. 
Impacto de la educación inicial en el rendimiento en primer grado de primaria...

\section{Resultados}

En la muestra de 304 estudiantes encontramos un $54.6 \%$ que pasaron por un CEI, $22.7 \%$ que pasaron por un PRONOEI y $22.6 \%$ que no tuvieron educación inicial de ningún tipo.

El análisis se realizó utilizando un modelo de regresión de tipo probit ordenado. El análisis permite identificar el impacto de cada una de las variables independientes (o regresores) en la variable dependiente, controlando estadísticamente por las demás (es decir el efecto de cada variable independiente que se presenta es la contribución única de esa variable en el contexto del modelo utilizado; véase el anexo A para mayores detalles).

Como se puede observar en el Cuadro $2,{ }^{10}$ los resultados muestran un efecto significativo tanto del paso por CEI como por PRONOEI en las dos variables dependientes (en ambos casos la significancia de los coeficientes es $p<0.05)^{11}$. Los resultados de las variables controladas concuerdan con lo que se esperaría de acuerdo a otros estudios: el nivel de hacinamiento tiene un efecto negativo (es decir a mayor hacinamiento en el hogar menor rendimiento en lenguaje y matemática) y el nivel educativo de la madre tiene un efecto positivo (a mayor educación de la madre mayor rendimiento del hijo en lenguaje y matemática, aunque debe señalarse que en el caso de matemática el efecto de esta variable es estadísticamente significativo sólo con $\mathrm{p}<0.10$ ). Los resultados por sexo muestran ligera ventaja para los hombres en lenguaje, aunque en ningún caso es significativo. El centro educativo del estudiante fue introducido como covariable para controlar por las diferencias asociadas al trato diferenciado a los estudiantes en cada centro (en este caso se contrasta el rendimiento de los estudiantes de cada centro educativo contra el del primer centro educativo).

10. Como se observa en el cuadro 2, las estimaciones se realizaron sobre una muestra de sólo 222 estudiantes. La reducción del número de casos fué resultado de la inclusión de los años de educación de la madre como variable independiente (explicativa) en el modelo, ya que la pregunta asociada a esta variable no fue completada en todos los cucstionarios de la encuesta. Sin embargo, los resultados se mantienen si esta variable es retirada y la estimación se realiza sobre todos aquéllos estudiantes con nota.

11. Los resultados de CEI y PRONOEI se contrastan con no tener educación inicial. 


\section{Cuadro 2}

Rendimiento en Matemática y Lenguaje (estimaciones del modelo probit ordenado, prueba $t$ en paréntesis)

\begin{tabular}{|c|c|c|}
\hline & Matematica & Lenguaje \\
\hline Asistió a un CEI (dummy) & $\begin{array}{l}0.574^{*} \\
(2.81)\end{array}$ & $\begin{array}{l}0.662 * \\
(3.21)\end{array}$ \\
\hline Asistió a un PRONOEI (dummy) & $\begin{array}{l}0.442^{* *} \\
(1.90)\end{array}$ & $\begin{array}{l}0.383^{* *} \\
(1.64)\end{array}$ \\
\hline Sexo (dummy para hombres) & $\begin{array}{r}0.001 \\
(0.01)\end{array}$ & $\begin{array}{c}0.058 \\
(0.36)\end{array}$ \\
\hline Años de educación de la madre & $\begin{array}{l}0.030^{* * *} \\
(1.30)\end{array}$ & $\begin{array}{l}0.023^{* * *} \\
(0.99)\end{array}$ \\
\hline Indice de hacinamiento & $\begin{array}{l}-0.158^{*} \\
-(2.72)\end{array}$ & $\begin{array}{l}-0.156^{*} \\
-(2.65)\end{array}$ \\
\hline Dummy colegio 2 & $\begin{array}{l}-0.068 \\
-(0.23)\end{array}$ & $\begin{array}{l}-0.271 \\
-(093)\end{array}$ \\
\hline Dummy colegio 3 & $\begin{array}{l}-0.139 \\
-(0.41)\end{array}$ & $\begin{array}{l}-0.195 \\
-(0.56)\end{array}$ \\
\hline Dummy colegio 4 & $\begin{array}{l}-0.607^{* *} \\
-(2.11)\end{array}$ & $\begin{array}{l}-0.784^{* *} \\
-(2.69)\end{array}$ \\
\hline Dummy colegio 5 & $\begin{array}{l}-0.069 \\
-(0.20)\end{array}$ & $\begin{array}{c}0.086 \\
(0.24)\end{array}$ \\
\hline Dummy colegio 6 & $\begin{array}{c}0.214 \\
(0.59)\end{array}$ & $\begin{array}{l}0.117 \\
(0.32)\end{array}$ \\
\hline Dummy colegio 7 & $\begin{array}{l}-0.284 \\
-(0.84)\end{array}$ & $\begin{array}{l}-0.096 \\
-(0.28)\end{array}$ \\
\hline Dummy colegio 8 & $\begin{array}{r}0.097 \\
(0.29)\end{array}$ & $\begin{array}{r}-0.037 \\
-(0.11)\end{array}$ \\
\hline Dummy colegio 9 & $\begin{array}{l}0.178 \\
(0.47)\end{array}$ & $\begin{array}{l}0.135 \\
(0.35)\end{array}$ \\
\hline $\begin{array}{l}\text { Parámetros adicionales: } \\
\mu 1\end{array}$ & $\begin{array}{r}-1.101 \\
(0.41)\end{array}$ & $\begin{array}{r}-1.036 \\
(0.42)\end{array}$ \\
\hline$\mu 2$ & $\begin{array}{l}0.183 \\
(0.40)\end{array}$ & $\begin{array}{r}0.064 \\
(0.41)\end{array}$ \\
\hline $\begin{array}{l}\text { Número de observaciones } \\
\text { Prueba chi2(12) } \\
\text { Probabilidad > chi2 }\end{array}$ & $\begin{array}{r}222 \\
33.27 \\
0.0016\end{array}$ & $\begin{array}{r}222 \\
39.42 \\
0.0002\end{array}$ \\
\hline
\end{tabular}

${ }^{*}$ p. $<0.01{ }^{* *}$ p. $<0.05{ }^{* * *}$ p. $<0.10$

84 
Impacto de la educación inicial en el rendimiento en primer grado de primaria...

Los resultados anteriores sin embargo no permiten discriminar el efecto específico del paso por el CEI o PRONOEI en la probabilidad de obtener la nota A (logró la competencia), B (en proceso de lograr la competencia) o C (no logró la competencia), sólo permiten decir que el efecto de estas variables es significativo estadísticamente.

El Cuadro 3 muestra el cambio en las probabilidades de obtener una A, B o C de acuerdo al tipo de educación inicial (nuevamente la comparación es con los estudiantes que no tuvieron educación inicial). Para este análisis se controló por las mismas variables mencionadas en el análisis del Cuadro 2.

Cuadro 3

Efecto Marginal de la Educación Inicial sobre la Probabilidad de obtener un Determinado Rendimiento

Matemática Lenguaje

CEI

No logró competencia (C)

En proceso (B)

$-12.7 \%$

$-15.6 \%$

Logró competencia (A)

$22.2 \%$

$-9.5 \%$

$25.1 \%$

\section{PRONOEI}

No logró competencia (C)

$-8.4 \%$

$-7.9 \%$

En proceso (B)

$-9.0 \%$

$-7.1 \%$

Logró competencia (A)
$15.0 \%$

Nota. Las cifras anteriores deben ser interpretadas como el cambio porcentual en las probabilidades de obtener una determinada nota, de modo que, cuando es positiva significa que la probabilidad aumenta y cuando es negativa la probabilidad disminuye.

El Cuadro 3 muestra que, tal cual se había señalado en la hipótesis general, el paso por la educación inicial (ya sea por CEI o PRONOEI) aumenta las probabilidades de obtener una mejor nota (A) y disminuye 
las probabilidades de obtener una peor nota (C). Por ejemplo, haber pasado por un CEI aumenta en $22 \%$ la probabilidad de obtener una A en matemática ( $y$ en $25 \%$ en lenguaje), mientras que reduce en $13 \%$ la probabilidad de obtener una C (16\% en el caso de lenguaje). Los resultados también confirman la hipótesis específica: las probabilidades asociadas a un mejor rendimiento son mayores en el caso del CEI que en el del PRONOEI. En el otro extremo, la probabilidad de tener una $\mathrm{C}$ es menor para los que pasaron por un CEI que para los que pasaron por un PRONOEI.

\section{Discusión}

A menudo se ha dicho que el problema de la educación primaria en el Perú ha dejado de ser la matrícula, dados los altos índices de matrícula de niños entre los 6 y 11 años (ver por ejemplo El Peruano, 1988). En cambio se argumenta que el problema es mejorar la calidad de la educación recibida (por ejemplo reducir las tasas de repetición y deserción, elevar el nivel de aprendizaje de los estudiantes en la escuela y mejorar la calidad de la interacción entre profesores y alumnos). Los resultados de este estudio sugieren que una forma de mejorar la calidad de la educación primaria es aumentar la cobertura de la educación inicial, y dentro de ella aquellos que han pasado por un Centro de Educación Inicial (CEI) tienen mejores resultados (al menos de acuerdo a las calificaciones asignadas por sus maestros) que aquellos que han pasado por los Programas No Escolarizados de Educación Inicial (PRONOEIS).

El presente estudio no presenta datos sobre la calidad de la educación ofrecida por los CEI y PRONOEI sino solo del rendimiento en primer grado de estudiantes que han pasado por estos centros. Sin embargo publicaciones anteriores (Myers, 1992) y conversaciones con el personal a cargo de supervisar los PRONOEI sugieren que se debería mejorar la calidad de la capacitación dada a las madres monitoras, la frecuencia y calidad de la supervisión a las madres monitoras, la cantidad y calidad de materiales educativos disponibles en los PRONOEI, y el tiempo en la tarea (es decir el número de horas que los alumnos pasan en los PRONOEI y 
Impacto de la educación inicial en el rendimiento en primer grado de primaria...

el aprovechamiento del mismo). Se debe destacar de todos modos que el funcionamiento de los PRONOEI (al igual que los CEI) no es uniforme, habiéndose observado algunos donde el funcionamiento y calidad parecían bastante superiores a los demás (de hecho como se dijo antes en algunos casos los PRONOEI están a cargo de madres animadoras y en otros de estudiantes de educación por que, de acuerdo a un especialista de una de las USE visitadas, "lo hacen mejor que las madres"). Esto sugiere una pregunta de investigación para un futuro estudio, y es identificar los factores del PRONOEI que están asociados a un mejor rendimiento de los estudiantes en su paso por la primaria.

Es importante destacar que el presente estudio se ha ocupado solamente de uno de los aspectos que debería ser influenciado por la educación inicial, y es un mejor aprovechamiento de la educación primaria. Otros aspectos que deberían ser influenciados por la educación inicial (por ejemplo la socialización, el desarrollo motor y el afectivo) deberían ser abordados en futuros estudios.

El presente estudio tampoco presenta datos sobre escuelas rurales, que ha sido una de las áreas donde más comúnmente se han instalado los PRONOEI. En el futuro sería sumamente interesante comparar el impacto de los PRONOEI en zonas rurales (el estudio de Myers, 1992, deja dudas sobre el impacto de estos; sin embargo los datos de ese estudio necesitan ser replicados).

Otra variable interesante para futuros estudios es la duración de la educación inicial. Es de esperar que a mayor número de años en educación inicial, mejor rendimiento en educación primaria. Esta es una hipótesis que debe responderse empíricamente (como se recordará, el Estado tiene planeado llegar al 100\% de los estudiantes entre 4 y 5 años para 2007; lo interesante en este caso sería determinar si dos años de educación inicial brindan significativamente mayores beneficios que uno y si tres brindan mayores beneficios que dos años de educación inicial). 
Se debe reiterar que los datos utilizados en el presente estudio fueron tomados retrospectivamente, recogiendo notas de "registros de notas" de los profesores para el año 1997. Este tipo de datos tiene las limitaciones de cualquier estudio que analiza los resultados en un solo punto del tiempo. En el futuro se debería planificar un estudio donde los niños sean seguidos desde los tres años en adelante, cubriendo al menos los primeros grados de educación primaria. El seguimiento de los niños en varios momentos de su desarrollo como estudiantes brindaría información valiosa en la medida en que este tipo de datos refleja aprendizaje, que es dinámico y no estático como los datos aquí presentados, y pueden ser vinculados a las experiencias del niño(a).

Entre los resultados de alguna manera inesperados del presente estudio está en primer lugar la ausencia de deserción en las escuelas estudiadas (todos aquellos matriculados en primer grado en 1997 continuaban en la escuela en 1998). Este resultado coincide con estadísticas oficiales y sugiere que el problema de la educación primaria no es la convocatoria de estudiantes en edad escolar sino, como se dijo antes, la calidad de la educación ofrecida. En segundo lugar la presencia de un $5 \%$ de alumnos repitentes, a pesar de existir una prohibición dada por el Ministerio de Educación, sugiere que el tratamiento de alumnos con bajo rendimiento requiere una redefinición por parte del Estado. Evidentemente el prohibir la repetición no es una solución para muchos profesores y directores del presente estudio, que piensan que promover a estudiantes a segundo grado aun cuando no hayan aprendido ciertas habilidades básicas ( $\mathrm{y}$ en un contexto donde hay solo un profesor para más de 40 alumnos) no ayuda a los estudiantes ni a los docentes. Seguramente será necesario en el futuro planificar medidas que complementen la prohibición de la repetición (por ejemplo se podría explorar la factibilidad de montar un sistema de recuperación a lo largo del año escolar o durante las vacaciones para aquellos estudiantes que se rezaguen respecto del grupo).

Los resultados del presente estudio sugieren que para mejorar la calidad de la educación primaria sería importante aumentar la cobertura de la educación inicial. Esta recomendación concuerda con estudios in- 
Impacto de la educación inicial en el rendimiento en primer grado de primaria...

ternacionales (por ejemplo Schiefelbein y Wolff, 1993). Al mismo tiempo, los resultados sugieren que el impacto de los PRONOEI es relativamente menor que el de los CEI y por lo tanto llaman a nuevos estudios aplicados que permitan detectar virtudes y deficiencias en los PRONOEI para mejorar las posibilidades de estos centros de afectar positivamente la vida de los niños que ahí se educan.

\section{Referencias}

Cueto, S., Jacoby, E. y Pollitt, E. (1997). Rendimiento de niños y niñas de zonas rurales y urbanas del Perú. Revista de Psicología de la Universidad Católica del Perú, 15(1), 115-1134.

Currie, J. y Thomas, D. (1998). School quality and the longer-term effects of Head Start. National Bureau of Economic Research, Working Paper Series 6362.

Data Social. Boletín de Difusión sobre políticas sociales (junio de 1998), IV(4). La educación inicial en el Perú. Lima: Instituto Apoyo.

INANDEP (1993). Tabulados del Censo Escolar 1993. Lima: Instituto de Estudios en Población y Desarrollo.

INEI (1994). Perú: Mapa de Necesidades Básicas Insatisfechas de los hogares a nivel distrital. Tomo I. Lima: Instituto Nacional de Estadística e Informática.

INEI (1995). Atraso y Deserción Escolar en Niños y Adolescentes. Lima: Instituto Nacional de Estadística e Informática.

Estadísticas de la Educación. (1998, setiembre 28). El Peruano, p. A5. Lockheed, M. y Verspoor, A. (1991). Improving Primary Education in Developing Countries. Washington DC: The World Bank.

Ministerio de Educación (1993). Censo Escolar. Lima: Autor.

Ministerio de Educación (1996). PRONOEI: Que aprendimos. Lima: Ministerio de Educación, UNICEF y Banco Interamericano de Desarrollo.

Ministerio de Educación (1997). Nueva Estructura del Sistema Educativo Peruano. Lima: Autor.

Myers, R. (1992). The Twelve who Survive. Londres: Routledge. 
Myers, R. (1996). Preschool education in Latin America: "State of the Practice" (Occasional paper series No 1). Washington DC: PREAL y CINDE.

Schiefelbein, E. (1990). Elementos para reflexionar sobre la calidad de la educación en América Latina (Informe de Investigación del Instituto Internacional de Planeamiento de la Educación No 86). París.

Schiefelbein, E. y Wolff, L. (1993). Repetición y rendimiento inadecuado en escuelas primarias De América Latina: Magnitudes, causas, relaciones y estrategias. Boletín Proyecto Principal de Educación en América Latina y el Caribe, 30, 17-50.

UNESCO (1995). '95 Statistical Yearbook. Lantham, MD: UNESCO/ Bernam Press.

\section{Anexo A}

Estimación de modelos Probit ordenados

Los modelos probit ordenados estiman la relación entre variables dependientes ordinales y un conjunto de variables independientes. A diferencia de un modelo probit estándar, la variable dependiente tiene más de dos posibles resultados, tratándose de variables categóricas cuyos resultados pueden ordenarse de menor a mayor, por ejemplo los niveles de rendimiento 'bajo', 'medio' y 'alto' tienen un orden claro de menor (obtener una C) a mayor (obtener una $\mathrm{A}$ ).

La estimación se realiza asumiendo que existe una variable latente no observada $y^{*}$ con función de distribución normal, que mantiene una relación con la variable categórica que sí se observa. Para los tres niveles de rendimiento escolar ( $\mathrm{C}$ si es bajo, $\mathrm{B}$ si es medio, A si es alto) la relación entre la variable categórica observada y y la variable latente $y^{*}$ se puede escribir como: 
Impacto de la educación inicial en el rendimiento en primer grado de primaria...

$$
\begin{aligned}
& y=C, y^{*}<\mu_{C}-\alpha X \\
& y=B, \mu_{C}-\alpha X<y^{*}<\mu_{B}-\alpha X \\
& y=A, y^{*}>\mu_{B}-\alpha X
\end{aligned}
$$

donde $X$ representa el conjunto de variables independientes, mientras que $\left(\alpha, \mu_{C}, \mu_{B}\right)$ son los parámetros a estimar. Los parámetros $(\alpha)$ son análogos a los de un probit estándar, mientras $\left(\mu_{C}, \mu_{B}\right)$ que corresponden a los límites que determinan la relación entre y la variable latente $y^{*}$.

Sin embargo, los coeficientes o parámetros $(\alpha)$ no reflejan directamente el efecto que un cambio en alguna variable independiente genera en la probabilidad de obtener un determinado rendimiento. Luego, es necesario calcular los "efectos marginales" de las variables independientes sobre cada una de las probabilidades. Los efectos marginales de cada una de las variables independientes se calculan - para cada una de las categorías- en base a los parámetros obtenídos en la estimación del probit ordenado y a los valores medios de todas las variables independientes (lo que se representa como $\bar{X}$ ), utilizando la fórmula:

$$
\frac{\left.\partial \operatorname{Pr}^{*} y=j\right)}{\partial x_{i}}=\left[\phi(\mu,-\alpha \bar{X})-\phi\left(\mu_{j-1}-\alpha \bar{X}\right)\right] \alpha_{i}(j=A, B, o \quad C)
$$

esta expresión ${ }^{12}$ refleja el efecto que la variable independiente $x_{i}$ tiene sobre la probabilidad de obtener el nivel de rendimiento $j$. Adicionalmente, debe tenerse presente que si la variable explicativa que se analiza es una dummy (por ejemplo, el haber pasado por un $\mathrm{CEI}^{13}$ ), la manera apropiada de obtener el efecto marginal sobre la probabilidad es calcular la diferencia entre las probabilidades cuando la dummy toma valor cero y cuando toma valor uno, manteniendo las demás variables en un valor constante (que usualmente es el valor medio).

12. La notación representa el valor de la probabilidad según la función normal estándar, evaluada en la expresión correspondiente.

13. Recuérdese que el paso por un CEI -o un PRONOEI- se representa aquí como una dummy que toma valor 1 si el alumno asistió a este tipo de institución y 0 si no lo hizo. 